\title{
EVALUATION OF SUNFLOWER GERMPLASMS FOR STEM BORER [Nupserha sp. near vexator (Pascoe)] RESISTANCE
}

\author{
Patil, B.V. ${ }^{*}$ and Jadhav, R.N.
}

Oilseeds Research Station, Latur-413 512 Maharashtra State, India

Received: July 09, 2007

Accepted: May 15, 2009

\section{SUMMARY}

As many as 251 insect and acarid species are known to attack sunflower crop worldwide. Recently, a stem borer has emerged as a new and regular pest of sunflower in Marathwada region (M.S.) India. It is a coleopterous grub Nupserha sp. near vexator (Pascoe), which belongs to the family Cerambycidae: Lamiinae. Hence, the screening of sunflower germplasm lines was conducted during last five years to identify resistance sources against stem borer for further exploitation in breeding programs. A set of 100 germplasm lines was annually received from All-India Coordinated Research Project on Sunflower during 2002-06 to evaluate sources of resistance for major sunflower pests. The screening work was carried out at Oilseeds Research Station, Latur, M.S., India, which was identified as a hotspot for stem borer in sunflower. Reactions to stem borer received from the germplasm lines were categorized for ease in evaluation of resistance potential of each test line. Stem borer incidence varied from free to $>80 \%$ in the germplasm under investigation during last five years indicating the presence of adequate variability in the material for their response to stem borer infestation.

Key words: sunflower, germplasm, stem borer, incidence, resistance

\section{INTRODUCTION}

Several species of both beneficial and harmful insects as well as microbial organisms are associated with sunflower (Helianthus annuus L.) crop. Among biotic constraints in sunflower production, insect pests and diseases are of major concern. Among harmful insects, seedling insect pests, capitulum borers and defoliators are considered as insect pests of economic importance. Among the defoliators, the tobacco caterpillar, the Bihar hairy caterpillar, the green semilooper and the cabbage green semilooper are of major importance. The army worm, the saf-

* Corresponding author: Ph/Fax: (off.)+91-02382-245294;

Resi:+91-02382-224939 M:+91-09421363306; e-mail: patilbvors@rediffmail.com 
flower caterpillar, the tea caterpillar, weevils and grasshoppers are the defoliators of major importance. The loss in seed yield due to defoliators in a rain-fed kharif crop was up to $268 \mathrm{~kg} / \mathrm{ha}$ recorded at Bangalore, (K.S.) India. Outbreaks of Spodoptera litura have been noticed in sunflower-growing areas of Karnatka and Maharashtra State of India. The capitulum borer (Helicoverpa armigera) is the most serious and destructive pest of all insect pests of sunflower (Anonymous ${ }^{1}$, 2006). As many as 251 insect and acarid species are known to attack sunflower crop worldwide (Rajamohan, 1976). Recently, a stem borer has emerged as a new pest of sunflower in Marathwada region (M.S.), India. It is a coleopterous grub, Nupserha sp. near vexator (Pascoe), which belongs to the family Cerambycidae: Lamiinae. Severe incidence up to $70 \%$ of stem borer was observed in farmers' fields during a survey of kharif sunflower in Latur district of Marathwada region (Anonymous, 1998). Incidence of stem borer in farmers' fields in July-sown crop was $42-55 \%$ whereas up to $25 \%$ incidence was recorded in August-sown crop during a pest survey in Marathwada region (Anonymous, 2001). Stem borer incidence was recorded to the extent $25 \%$ at Ambulga in Latur district (Anonymous, 2004) and $10-25 \%$ incidence was reported for most of the locations of Latur district (Anonymous $^{2}$, 2006). Therefore, germplasm sunflower lines were screening during last five years to identify resistance sources against stem borer for further exploitation in breeding programs.

\section{MATERIAL AND METHODS}

A set of 100 germplasm lines from All-India Coordinated Research Project on Sunflower was received per year during 2002-06 to evaluate sources of resistance for major pests of sunflower. The screening work was carried out at Oilseeds Research Station, Latur, M.S., India, which was identified as a hotspot for stem borer in sunflower. In order to generate information on potential donors for stem borer resistance, the present study was conducted during 2002-06. A total of 500 germplasm lines have been screened in the last five years. One hundred test lines were sown per year in kharif season (July-August sowing) in a single row of $4.2 \mathrm{~m}$ length with the spacing of $60 \times 30 \mathrm{~cm}$. All recommended agronomic practices were followed. Observations were made at the time of harvest by cutting the stem at the collar region. The percent incidence was worked out on the basis of the number of plants infested by stem borer in each test line. The data generated during the last five years are presented in Tables 1 to 5 .

\section{RESULTS}

Reactions to the stem borer ( $N$. vexator) in sunflower germplasm lines were categorized for ease in evaluation of resistance potential of each test line. The lines that showed zero reaction to the stem borer were categorized as highly resistant, the 
Table 1: Germplasm lines tested during Table 2: Germplasm lines tested during kharif, 2002

\begin{tabular}{|c|c|c|c|c|c|c|c|}
\hline Entry & $\begin{array}{l}\text { \% SB inci- } \\
\text { dence }\end{array}$ & Entry & $\begin{array}{c}\text { \% SB inci- } \\
\text { dence }\end{array}$ & Entry & $\begin{array}{l}\text { \% SB inci- } \\
\text { dence }\end{array}$ & Entry & $\begin{array}{c}\text { \% SB inci- } \\
\text { dence }\end{array}$ \\
\hline GMU-01 & 18.0 & GMU-51 & 25.0 & GMU-101 & 16.6 & GMU-151 & 15.3 \\
\hline GMU-02 & 33.0 & GMU-52 & 36.0 & GMU-102 & 30.7 & GMU-152 & 21.4 \\
\hline GMU-03 & 18.0 & GMU-53 & 50.0 & GMU-103 & 27.2 & GMU-153 & 18.1 \\
\hline GMU-04 & 8.0 & GMU-54 & 33.0 & GMU-104 & 28.5 & GMU-154 & 21.4 \\
\hline GMU-05 & 20.0 & GMU-55 & 54.0 & GMU-105 & 7.1 & GMU-155 & 28.5 \\
\hline GMU-06 & 25.0 & GMU-56 & 50.0 & GMU-106 & 14.2 & GMU-156 & 27.2 \\
\hline GMU-07 & 33.0 & GMU-57 & 33.0 & GMU-107 & 21.4 & GMU-157 & 38.4 \\
\hline GMU-08 & 25.0 & GMU-58 & 22.0 & GMU-108 & 14.2 & GMU-158 & 14.2 \\
\hline GMU-09 & 36.0 & GMU-59 & 36.0 & GMU-109 & 28.5 & GMU-159 & 7.6 \\
\hline GMU-10 & 33.0 & GMU-60 & 50.0 & GMU-110 & 14.2 & GMU-160 & 28.5 \\
\hline GMU-11 & 20.0 & GMU-61 & 27.0 & GMU-111 & 25.0 & GMU-161 & 15.3 \\
\hline GMU-12 & 40.0 & GMU-62 & 42.0 & GMU-112 & 15.3 & GMU-162 & 7.6 \\
\hline GMU-13 & 25.0 & GMU-63 & 30.0 & GMU-113 & 8.3 & GMU-163 & 0.0 \\
\hline GMU-14 & 33.0 & GMU-64 & 22.0 & GMU-114 & 14.2 & GMU-164 & 18.1 \\
\hline GMU-15 & 42.0 & GMU-65 & 27.0 & GMU-115 & 8.3 & GMU-165 & 21.4 \\
\hline GMU-16 & 27.0 & GMU-66 & 25.0 & GMU-116 & 7.6 & GMU-166 & 9.0 \\
\hline GMU-17 & 50.0 & GMU-67 & 33.0 & GMU-117 & 21.4 & GMU-167 & 28.5 \\
\hline GMU-18 & 29.0 & GMU-68 & 50.0 & GMU-118 & 15.3 & GMU-168 & 15.3 \\
\hline GMU-19 & 0.0 & GMU-69 & 36.0 & GMU-119 & 14.2 & GMU-169 & 25.0 \\
\hline GMU-20 & 33.0 & GMU-70 & 50.0 & GMU-120 & 14.2 & GMU-170 & 33.3 \\
\hline GMU-21 & 27.0 & GMU-71 & 11.0 & GMU-121 & 14.2 & GMU-171 & 14.2 \\
\hline GMU-22 & 22.0 & GMU-72 & 18.0 & GMU-122 & 21.4 & GMU-172 & 16.6 \\
\hline GMU-23 & 13.0 & GMU-73 & 33.0 & GMU-123 & 15.3 & GMU-173 & 21.4 \\
\hline GMU-24 & 18.0 & GMU-74 & 18.0 & GMU-124 & 45.4 & GMU-174 & 15.3 \\
\hline GMU-25 & 25.0 & GMU-75 & 33.0 & GMU-125 & 0.0 & GMU-175 & 25.0 \\
\hline GMU-26 & 18.0 & GMU-76 & 25.0 & GMU-126 & 33.3 & GMU-176 & 28.5 \\
\hline GMU-27 & 11.0 & GMU-77 & 36.0 & GMU-127 & 14.2 & GMU-177 & 16.6 \\
\hline GMU-28 & 10.0 & GMU-78 & 40.0 & GMU-128 & 50.0 & GMU-178 & 8.3 \\
\hline GMU-29 & 36.0 & GMU-79 & 33.0 & GMU-129 & 16.6 & GMU-179 & 23.0 \\
\hline GMU-30 & 30.0 & GMU-80 & 44.0 & GMU-130 & 20.0 & GMU-180 & 16.6 \\
\hline GMU-31 & 33.0 & GMU-81 & 38.0 & GMU-131 & 36.3 & GMU-181 & 7.6 \\
\hline GMU-32 & 25.0 & GMU-82 & 27.0 & GMU-132 & 16.6 & GMU-182 & 21.4 \\
\hline GMU-33 & 18.0 & GMU-83 & 42.0 & GMU-133 & 23.0 & GMU-183 & 16.6 \\
\hline GMU-34 & 25.0 & GMU-84 & 36.0 & GMU-134 & 16.6 & GMU-184 & 15.3 \\
\hline GMU-35 & 33.0 & GMU-85 & 33.0 & GMU-135 & 20.0 & GMU-185 & 21.4 \\
\hline GMU-36 & 33.0 & GMU-86 & 22.0 & GMU-136 & 14.2 & GMU-186 & 30.7 \\
\hline GMU-37 & 33.0 & GMU-87 & 18.0 & GMU-137 & 16.6 & GMU-187 & 21.4 \\
\hline GMU-38 & 18.0 & GMU-88 & 0.0 & GMU-138 & 25.0 & GMU-188 & 14.2 \\
\hline GMU-39 & 33.0 & GMU-89 & 50.0 & GMU-139 & 23.0 & GMU-189 & 21.4 \\
\hline GMU-40 & 54.0 & GMU-90 & 33.0 & GMU-140 & 25.0 & GMU-190 & 28.5 \\
\hline GMU-41 & 25.0 & GMU-91 & 27.0 & GMU-141 & 14.2 & GMU-191 & 15.3 \\
\hline GMU-42 & 18.0 & GMU-92 & 44.0 & GMU-142 & 8.3 & GMU-192 & 15.3 \\
\hline GMU-43 & 27.0 & GMU-93 & 45.0 & GMU-143 & 36.3 & GMU-193 & 25.0 \\
\hline GMU-44 & 25.0 & GMU-94 & 38.0 & GMU-144 & 20.3 & GMU-194 & 30.7 \\
\hline GMU-45 & 33.0 & GMU-95 & 33.0 & GMU-145 & 45.4 & GMU-195 & 23.0 \\
\hline GMU-46 & 36.0 & GMU-96 & 42.0 & GMU-146 & 18.1 & GMU-196 & 14.2 \\
\hline GMU-47 & 30.0 & GMU-97 & 36.0 & GMU-147 & 15.3 & GMU-197 & 35.7 \\
\hline GMU-48 & 45.0 & GMU-98 & 0.0 & GMU-148 & 18.1 & GMU-198 & 28.5 \\
\hline GMU-49 & 55.0 & GMU-99 & 10.0 & GMU-149 & 28.6 & GMU-199 & 15.3 \\
\hline GMU-50 & 36.0 & GMU-100 & 18.0 & GMU-150 & 25.0 & GMU-200 & 23.0 \\
\hline
\end{tabular}


incidence $<10 \%$ was categorized as resistant, $<15 \%$ as moderately resistant, $<20 \%$ as low resistant, $<30 \%$ as tolerant, between 30 and $50 \%$ to be discourage for further exploitation and the incidence $>50 \%$ was treated as susceptible. The response of set I of germplasm lines presented in Table 1 revealed that GMU-19, 88 and 98 were highly resistant, GMU-04, 28 and 99 were resistant, GMU-23, 27 and 71 were moderately resistant, whereas GMU-01, 03, 24, 26, 33, 42, 72, 74, $87 \& 100$ were evaluated as low resistant to stem borer. The following 25 lines were found to be tolerant: GMU-06, 08, 13, 16, 18, 21, 22, 25, 30, 32, 34, 41, 43, 44, 47, 51, 58, 61, $64,65,66,76,82,86$ and 91 , and the following 9 lines showed susceptible reactions: GMU 17, 49, 53, 55, 56, 60, 68, 70 and 89. Set II of 100 germplasm lines screened during kharif 2003 against stem borer (Table 2) showed that 34 lines were tolerant to the stem borer, GMU-103, 104, 107, 109, 114, 117, 122, 133, 138, $139,140,149,150,152,154,155,156,160,165,167,169,173,175,176,179$, $182,185,187,189,190,193,195,198$ and 200, 26 lines were evaluated as low resistant to the stem borer, GMU-112, 118, 123, 129, 130, 132, 134, 135, 137, $146,147,148,151,153,161,164,168,172,174,177,180,183,184,191,192$ and 199. Twelve lines were moderately resistant, GMU-110, 114, 119, 120, 121 , 127, 136, 141, 158, 171, 188 and 196. Nine lines were resistant, GMU-113, 115, $116,142,159,162,166,178$ and 181, whereas GMU-125 and 163 were evaluated as highly resistant. GMU-128 was the only line that recorded high incidence of the pest, to the extent of $50 \%$. Set III of sunflower germplasm was tested in 2004 . The data presented in Table 3 show that GMU 265, 279 and 288 were highly resistant to the stem borer but it is interesting that no entry emerged as resistant. GMU-256 and 299 showed moderate resistance to the stem borer whereas GMU $245 \& 273$ were low resistant. Eleven lines, GMU 215, 226, 228, 230, 239, 258, 262, 275, 276 , 280 and 292, were tolerant to the stem borer. The highest incidence among the 100 lines, $>70 \%$, was recorded in GMU-274, 285 and 286. Set IV of sunflower germplasm was sown in kharif, 2005 and the results reported in Table 4 indicate that the incidence of stem borer reached its peak in that year, $>80 \%$. The highest incidence was recorded in GMU 351, 358 and 360. On the other hand, GMU-400 showed zero reaction to stem borer. GMU-335, 369 and 373 showed resistance to the stem borer while GMU-304, 312, 328 and 389 were moderately resistant. GMU327, 357 and 394 were in the low resistant category and GMU- 310, 313, 314, 315, 318, 320, 321, 322, 329, 333, 338, 345, 347, 348, 352, 355, 364, 376, 376, 377, $381,392,393,398$ and 399 were in the tolerant category. Set V of sunflower germplasm was screened during kharif, 2006 and data generated and presented in Table 5 show that the incidence of the stem borer was in the range from free to $50 \%$. Of the 100 lines tested, GMU 459, 480 and 488 were highly resistant to the stem borer as they remained totally free from infestation, while GMU 500 was recorded as having high incidence of the pest, to the extent if 50\%. Among the 100 lines tested, 6 were resistant, GMU 431, 439, 444, 453, 464 and 482, 14 were moderately resistant, GMU-410, 433, 438, 445, 452, 457, 458, 465, 472, 475, 483, 
Table 3: Germplasm lines tested during Table 4: Germplasm lines tested during kharif, 2004

\begin{tabular}{|c|c|c|c|c|c|c|c|}
\hline Entry & $\begin{array}{c}\% \text { SB } \\
\text { incidence }\end{array}$ & Entry & $\begin{array}{c}\% \text { SB } \\
\text { incidence }\end{array}$ & Entry & $\begin{array}{c}\% \text { SB } \\
\text { incidence }\end{array}$ & Entry & $\begin{array}{c}\text { \% SB } \\
\text { incidence }\end{array}$ \\
\hline GMU-201 & 38.0 & GMU-251 & 55.0 & GMU-301 & 50.0 & GMU-351 & 80.0 \\
\hline GMU-202 & 33.0 & GMU-252 & 55.0 & GMU-302 & 73.0 & GMU-352 & 30.0 \\
\hline GMU-203 & 40.0 & GMU-253 & 33.0 & GMU-303 & 33.0 & GMU-353 & 60.0 \\
\hline GMU-204 & 33.0 & GMU-254 & 40.0 & GMU-304 & 11.0 & GMU-354 & 64.0 \\
\hline GMU-205 & $P G$ & GMU-255 & 43.0 & GMU-305 & 44.0 & GMU-355 & 25.0 \\
\hline GMU-206 & 45.0 & GMU-256 & 14.0 & GMU-306 & 44.0 & GMU-356 & 45.0 \\
\hline GMU-207 & 57.0 & GMU-257 & 40.0 & GMU-307 & 42.0 & GMU-357 & 20.0 \\
\hline GMU-208 & 63.0 & GMU-258 & 25.0 & GMU-308 & 31.0 & GMU-358 & 80.0 \\
\hline GMU-209 & 38.0 & GMU-259 & 33.0 & GMU-309 & 50.0 & GMU-359 & 36.0 \\
\hline GMU-210 & 50.0 & GMU-260 & 50.0 & GMU-310 & 22.0 & GMU-360 & 80.0 \\
\hline GMU-211 & 42.0 & GMU-261 & 33.0 & GMU-311 & 50.0 & GMU-361 & 50.0 \\
\hline GMU-212 & 40.0 & GMU-262 & 29.0 & GMU-312 & 14.0 & GMU-362 & 66.0 \\
\hline GMU-213 & 33.0 & GMU-263 & 52.0 & GMU-313 & 30.0 & GMU-363 & 66.0 \\
\hline GMU-214 & 50.0 & GMU-264 & 33.0 & GMU-314 & 25.0 & GMU-364 & 25.0 \\
\hline GMU-215 & 25.0 & GMU-265 & 0.0 & GMU-315 & 22.0 & GMU-365 & \\
\hline GMU-216 & 43.0 & GMU-266 & 57.0 & GMU-316 & 36.0 & GMU-366 & 66.0 \\
\hline GMU-217 & 50.0 & GMU-267 & 38.0 & GMU-317 & 33.0 & GMU-367 & 43.0 \\
\hline GMU-218 & 40.0 & GMU-268 & 67.0 & GMU-318 & 29.0 & GMU-368 & 50.0 \\
\hline GMU-219 & 33.0 & GMU-269 & 50.0 & GMU-319 & 45.0 & GMU-369 & 10.0 \\
\hline GMU-220 & 63.0 & GMU-270 & 50.0 & GMU-320 & 25.0 & GMU-370 & 54.0 \\
\hline GMU-221 & 50.0 & GMU-271 & 60.0 & GMU-321 & 30.0 & GMU-371 & 58.0 \\
\hline GMU-222 & 66.0 & GMU-272 & 50.0 & GMU-322 & 27.0 & GMU-372 & 60.0 \\
\hline GMU-223 & 50.0 & GMU-273 & 17.0 & GMU-323 & 45.0 & GMU-373 & 10.0 \\
\hline GMU-224 & 50.0 & GMU-274 & 71.0 & GMU-324 & 55.0 & GMU-374 & $P G$ \\
\hline GMU-225 & 40.0 & GMU-275 & 22.0 & GMU-325 & 32.0 & GMU-375 & 54.0 \\
\hline GMU-226 & 22.0 & GMU-276 & 28.0 & GMU-326 & 60.0 & GMU-376 & 25.0 \\
\hline GMU-227 & 38.0 & GMU-277 & 44.0 & GMU-327 & 20.0 & GMU-377 & 25.0 \\
\hline GMU-228 & 22.0 & GMU-278 & 44.0 & GMU-328 & 15.0 & GMU-378 & 31.0 \\
\hline GMU-229 & 44.0 & GMU-279 & 0.0 & GMU-329 & 25.0 & GMU-379 & 50.0 \\
\hline GMU-230 & 25.0 & GMU-280 & 25.0 & GMU-330 & 52.0 & GMU-380 & $P G$ \\
\hline GMU-231 & 50.0 & GMU-281 & 66.0 & GMU-331 & 35.0 & GMU-381 & 22.0 \\
\hline GMU-232 & 40.0 & GMU-282 & 63.0 & GMU-332 & 30.0 & GMU-382 & 54.0 \\
\hline GMU-233 & 66.0 & GMU-283 & 66.0 & GMU-333 & 29.0 & GMU-383 & $P G$ \\
\hline GMU-234 & 50.0 & GMU-284 & 57.0 & GMU-334 & 45.0 & GMU-384 & 45.0 \\
\hline GMU-235 & 50.0 & GMU-285 & 75.0 & GMU-335 & 9.0 & GMU-385 & 73.0 \\
\hline GMU-236 & 66.0 & GMU-286 & 77.0 & GMU-336 & 38.0 & GMU-386 & 45.0 \\
\hline GMU-237 & 45.0 & GMU-287 & 50.0 & GMU-337 & 46.0 & GMU-387 & 60.0 \\
\hline GMU-238 & 44.0 & GMU-288 & 0.0 & GMU-338 & 29.0 & GMU-388 & 38.0 \\
\hline GMU-239 & 29.0 & GMU-289 & 43.0 & GMU-339 & 64.0 & GMU-389 & 14.0 \\
\hline GMU-240 & 60.0 & GMU-290 & 38.0 & GMU-340 & 55.0 & GMU-390 & 42.0 \\
\hline GMU-241 & 66.0 & GMU-291 & 50.0 & GMU-341 & 40.0 & GMU-391 & 38.0 \\
\hline GMU-242 & 45.0 & GMU-292 & 30.0 & GMU-342 & 38.0 & GMU-392 & 27.0 \\
\hline GMU-243 & 50.0 & GMU-293 & 50.0 & GMU-343 & 50.0 & GMU-393 & 25.0 \\
\hline GMU-244 & 40.0 & GMU-294 & 45.0 & GMU-344 & 56.0 & GMU-394 & 20.0 \\
\hline GMU-245 & 20.0 & GMU-295 & 50.0 & GMU-345 & 22.0 & GMU-395 & 36.0 \\
\hline GMU-246 & 50.0 & GMU-296 & 50.0 & GMU-346 & 57.0 & GMU-396 & 33.0 \\
\hline GMU-247 & 55.0 & GMU-297 & 33.0 & GMU-347 & 25.0 & GMU-397 & 33.0 \\
\hline GMU-248 & 38.0 & GMU-298 & 57.0 & GMU-348 & 25.0 & GMU-398 & 25.0 \\
\hline GMU-249 & 63.0 & GMU-299 & 14.0 & GMU-349 & 60.0 & GMU-399 & 29.0 \\
\hline GMU-250 & 57.0 & GMU-300 & 33.0 & GMU-350 & 31.0 & GMU-400 & 0.0 \\
\hline
\end{tabular}


484, 494 and 495, and 15 lines showed low resistance to the stem borer, GMU-401, $408,412,426,435,440,441,474,477,481,485,487,489,490$ and 493. The tolerant category included 14 lines, GMU-402, 403, 409, 414, 436, 451, 454, 456, 461, 462, 463, 473, 478 and 499.

Table 5: Germplasm lines tested during kharif, 2006

\begin{tabular}{|c|c|c|c|c|c|c|c|}
\hline Entry & $\begin{array}{c}\% \text { SB } \\
\text { incidence }\end{array}$ & Entry & $\begin{array}{c}\% \text { SB } \\
\text { incidence }\end{array}$ & Entry & $\begin{array}{c}\% \text { SB } \\
\text { incidence }\end{array}$ & Entry & $\begin{array}{c}\% \text { SB } \\
\text { incidence }\end{array}$ \\
\hline GMU-401 & 16.6 & GMU-426 & DS & GMU-451 & 22.2 & GMU-476 & 33.0 \\
\hline GMU-402 & 25.0 & GMU-427 & DS & GMU-452 & 11.0 & GMU-477 & 20.0 \\
\hline GMU-403 & 28.6 & GMU-428 & 20 & GMU-453 & 9.1 & GMU-478 & 23.1 \\
\hline GMU-404 & $P G$ & GMU-429 & DS & GMU-454 & 30.0 & GMU-479 & 33.0 \\
\hline GMU-405 & 41.6 & GMU-430 & DS & GMU-455 & 33.2 & GMU-480 & 0.0 \\
\hline GMU-406 & $P G$ & GMU-431 & 8.3 & GMU-456 & 22.2 & GMU-481 & 18.0 \\
\hline GMU-407 & $P G$ & GMU-432 & DS & GMU-457 & 12.5 & GMU-482 & 10.0 \\
\hline GMU-408 & 16.6 & GMU-433 & 14.3 & GMU-458 & 11.0 & GMU-483 & 11.0 \\
\hline GMU-409 & 28.5 & GMU-434 & $P G$ & GMU-459 & 0.0 & GMU-484 & 14.3 \\
\hline GMU-410 & 14.3 & GMU-435 & 20.0 & GMU-460 & DS & GMU-485 & 16.6 \\
\hline GMU-411 & $P G$ & GMU-436 & 28.5 & GMU-461 & 23.1 & GMU-486 & 37.5 \\
\hline GMU-412 & 16.6 & GMU-437 & DS & GMU-462 & 25.0 & GMU-487 & 20.0 \\
\hline GMU-413 & $P G$ & GMU-438 & 11.0 & GMU-463 & 25.0 & GMU-488 & 0.0 \\
\hline GMU-414 & 25.0 & GMU-439 & 10.0 & GMU-464 & 9.1 & GMU-489 & 16.6 \\
\hline GMU-415 & $P G$ & GMU-440 & 20.0 & GMU-465 & 14.3 & GMU-490 & 20.0 \\
\hline GMU-416 & $P G$ & GMU-441 & 20.0 & GMU-466 & DS & GMU-491 & - \\
\hline GMU-417 & $P G$ & GMU-442 & DS & GMU-467 & DS & GMU-492 & 33.0 \\
\hline GMU-418 & $P G$ & GMU-443 & DS & GMU-468 & DS & GMU-493 & 16.6 \\
\hline GMU-419 & $P G$ & GMU-444 & 10.0 & GMU-469 & DS & GMU-494 & 14.3 \\
\hline GMU-420 & $P G$ & GMU-445 & 14.3 & GMU-470 & DS & GMU-495 & 11.0 \\
\hline GMU-421 & $P G$ & GMU-446 & DS & GMU-471 & DS & GMU-496 & 40.0 \\
\hline GMU-422 & DS & GMU-447 & DS & GMU-472 & 11.0 & GMU-497 & DS \\
\hline GMU-423 & DS & GMU-448 & DS & GMU-473 & 27.3 & GMU-498 & DS \\
\hline GMU-424 & DS & GMU-449 & DS & GMU-474 & 16.6 & GMU-499 & 28.6 \\
\hline GMU-425 & DS & GMU-450 & DS & GMU-475 & 12.5 & GMU-500 & 50.0 \\
\hline
\end{tabular}

SB-stem borer, PG-poor germination,

DS-disease susceptible (wiped out with disease either by downy mildew, necrosis or both)

\section{DISCUSSION}

Stem borer incidence varied from free to $>80 \%$ in the germplasm under investigation during last five years indicating the presence of adequate variability in the material for their response to stem borer infestation. Anonymous (2001) reported that out of 50 germplasm lines of sunflower screened for stem borer resistance, 22 were found free from stem borer, one line, Acc. No. 210, recorded the incidence $<10 \%$, nine entries showed the incidence $<15 \%, 13$ lines recorded the incidence 
below $20 \%, 11$ lines showed tolerance to stem borer, i.e., the incidence $<30 \%$ and 5 lines were $100 \%$ susceptible to the stem borer. Anonymous (2002) reported that among 96 sunflower germplasm lines evaluated for stem borer resistance, 12 lines showed resistance to the stem borer, i.e., the incidence $<10 \%$, 30 lines were $<20 \%$ and 19 lines were infected below 30\%. Three lines registered the incidence of stem borer to the extent $60 \%$.

\title{
ACKNOWLEDGEMENTS
}

Authors are grateful to Director of Research, MAU, Parbhani, and Project Director, DOR, Hyderabad, for providing funds and facilities which enabled us to conduct the experiments.

\section{REFERENCES}

Anonymous, 1998. Annual Progress Report. All India Research Project on Sunflower, DOR, Hyderabad, India, pp. 168.

Anonymous, 2001. Annual Progress Report. All India Research Project on Sunflower, DOR, Hyderabad, India, pp. 142-150.

Anonymous, 2002. Annual Progress Report. All India Research Project on Sunflower, DOR, Hyderabad, India, pp. 161-164.

Anonymous, 2004. Annual Progress Report. All India Research Project on Sunflower, DOR, Hyderabad, India, pp. 156.

Anonymous $^{2}, 2006$. Annual Progress Report. All India Research Project on Sunflower, DOR, Hyderabad, India, pp. 146-163.

Anonymous ${ }^{1}, 2006$. Research Achievements in Sunflower. All-India Research Project on Sunflower, DOR and Hyderabad, pp. 55-57.

Rajamohan, N., 1976. Pest complex of sunflower a bibliography. PANS, 22: 546-563.

\section{EVALUACIÓN DE GERMOPLASMA DE GIRASOL POR RESISTENCIA A BARRENADOR DEL TALLO [(Nupserha sp. near vexator (Pascoe)]}

\author{
RESUMEN
}

\begin{abstract}
A lo largo del mundo, existen 251 especies de insectos ácaros que se sabe atacan al girasol. Actualmente el barrenador del tallo emerge como una peste nueva y regular para girasol en la región de Marathwada (M.S.) India. Es una larva de coleóptero perteneciente a la especie Nupserha sp. near vexator (Pascoe) y pertenece a la familia Cerambycidae: Lamiinae. Durante los últimos cinco años se evaluaron líneas de girasol con el objetivo de identificar fuentes de resistencia al barrenador del tallo para su posterior utilización en programas de mejoramiento. Durante los años 2002/2006 se recibieron cien líneas del programa de investigación coordinado All India para evaluar fuentes de resistencia a las principales pestes del girasol. El trabajo de evaluación se llevó a cabo en la Oilseeds Research Station, Latur, M.S., India, identificado como un ambiente de alta infestación del barrenador del tallo de girasol. Las reacciones recibidas de las líneas contra el barrenador del tallo se categorizaron por su facilidad de evaluación del potencial de resistencia de cada línea. La incidencia del barrenador del tallo varió desde cero ataque a más de $80 \%$ en el
\end{abstract}


germoplasma bajo investigación durante los cinco años, lo que indica la presencia de variabilidad adecuada en este material en términos de su respuesta a la infestación con barrenador del tallo.

\section{ÉVALUATION DE LA RÉSISTANCE DU GERMOPLASME DE TOURNESOL À LA SORTE D'INSECTE Nupserha sp. near vexator (Pascoe)}

\section{RÉSUMÉ}

Au moins 251 espèces d' insectes ou d' acariens sont connus pour attaquer le tournesol dans le monde. Actuellement, une chenille foreuse de la tige apparaît de façon régulière comme un insecte nuisible dans la région de Marathwada [M.S., Inde). Il s'agit d'un coléoptère proche de Nupserha vexator (Pascoe) appartenant à la famille des Cerambycidae: Lamiinae. Le screening de lignées de tournesol a été conduit au cours des cinq dernières années pour identifier des sources de résistance à exploiter ultérieurement dans les programmes d'amélioration génétique. Un ensemble de 100 lignées du programme coordonné de recherche pan-indien ont été reçu annuellement de 2002 à 2006 pour évaluer les sources de résistance aux principaux insectes nuisibles. Le travail de screening a été conduit à la Station de Recherche sur le Oléagineux de Latur [M.S. Inde] qui a été identifiée comme un point chaud pour la chenille foreuse de tige du tournesol. Les réactions des lignées ont été classifiées du niveau "sain" au niveau "plus de $80 \%$ d'attaque", ce qui indique la présence d'une variabilité pour la réponse aux attaques de la chenille foreuse de tige. 